\title{
Precauciones del aislamiento de los pacientes con enfermedades transmisibles en una institución de tercer nivel
}

\author{
Gloria Ortiz-López, * Ricarda Miranda-Tinoco,* María Guadalupe Lara-Martínez**
}

\begin{abstract}
RESUMEN
El concepto de aislamiento para personas con enfermedades transmisibles fue aplicado desde tiempos bíblicos, una muestra de ello son las colonias de leprosos. ${ }^{1}$ Durante la guerra de Crimea, Florence Nightingal concluyó en la necesidad de dividir las salas comunes en cubículos y enfatizó la importancia de la asepsia y del mantener los ambientes limpios, cambiando así el concepto de la transmisión de las infecciones. ${ }^{2}$ Con estos ejemplos, podemos ver a través de la historia como los conceptos y procedimientos se van mejorando y avanzando con el propósito de controlar las infecciones. En 1996, el Comité Asesor de Prácticas sobre el Control de Infecciones Hospitalarias (HICPAC) publicó la Guía para las Precauciones de Aislamiento en los Hospitales, definiendo con ello las precauciones estándar y las precauciones basadas en la transmisión. ${ }^{3}$ El objetivo de este trabajo fue hacer una revisión bibliográfica de los diferentes artículos científicos sobre las precauciones del manejo de pacientes con enfermedades transmisibles en una institución de tercer nivel para comprobar si existe o no una estandarización en las recomendaciones y en los procedimientos de enfermería en la práctica diaria. Se enfocó en las precauciones del aislamiento de los pacientes con enfermedades transmisibles mediante la revisión exhaustiva de diversas fuentes bibliográficas y mediante de fuentes electrónicas. Como resultado de dicha revisión comprobamos que sí existe una estandarización en las recomendaciones para el aislamiento del enfermo infeccioso, sin embargo, con la revisión de los manuales de diferentes instituciones observamos que hace falta ser más específico en los procedimientos, uso y manejo del material requerido para cumplir con las recomendaciones para el aislamiento, ya que un número importante de estos manuales no cuenta con estos datos, así como la disponibilidad y difusión de éstos.
\end{abstract}

Palabras clave: Transmisión, infección, precauciones, aislamiento.

\section{Isolation precautions in patients with communicable diseases in a third-level institution}

\begin{abstract}
The concept of isolation for people with contagious diseases, was applied since biblical times, a sample are leper colonies, during the Crimean ar Florence Nightingale concludes the need to divide the communal rooms in cubicles and emphasizes the importance of asepsis and maintain clean environments, so changing the concept of the transmission of infections. With these examples we can see through the history as the concepts and procedures are getting better and advancing in order to control infections. In 1996, the Advisory Committee for Practice on Control of Hospital Infections (HICPAC) published the Guidelines for Isolation Precautions in Hospitals defining for it the standard precautions and precautions based on transmission. The objective of this study was to review the literature of different scientific articles about precautions for handling patients with contagious diseases in a third level institution, to verify whether or not standardization exists in the
\end{abstract}

* Enfermera General. Instituto Nacional de Neurología y Neurocirugía MVS. Adscrita al Servicio de Neuroinfectología ** Enfermera General, Instituto Nacional de Neurología y Neurocirugía MVS. Adscrita al Servicio de Neurocirugía

Correspondencia: Enfermera Gral. Gloria Ortiz López. Tel: 57583042, cel: 5551820856. E-mail: ortizgloria52@hotmail.com

Este artículo puede ser consultado en versión completa en http://www.medigraphic.com/enfermerianeurologica 
recommendations and procedures in nursing daily practice. It focused on isolation precautions for patients with contagious diseases through an exhaustive review of various literature sources through electronic sources. As a result of that review we check that there is standardization in the recommendations for isolation of infectious patient. However, with the revision of textbooks from different institutions, we see that need to be more specific in the procedures, use and handling of material required to comply with the recommendations for isolation because a significant number of these manuals do not have these data. Well as availability and dissemination of these.

Key words: Transmission, infection, precautions, insulation.

\section{INTRODUCCIÓN}

E xisten recomendaciones universales para el aislamiento de pacientes hospitalizados con enfermedades transmisibles, sin embargo, en la práctica diaria se ha observado que existen discrepancias en el personal de salud al aplicar los procedimientos para cumplir estas recomendaciones, lo que podría influir en el índice de infecciones nosocomiales y en los accidentes laborales.

A través de la historia los conceptos y procedimientos para el aislamiento de los pacientes con enfermedades transmisibles se han ido modificando con la finalidad de reducir el índice de infecciones nosocomiales, sin embargo, a partir de la experiencia en la práctica diaria se han detectado diferencias en la aplicación de los procedimientos de aislamientos. Por lo anterior, el objetivo de esta revisión bibliográfica fue hacer un análisis sobre las precauciones para el manejo de pacientes con enfermedades transmisibles en una institución de tercer nivel, basadas en las normatividad oficial vigente. El objetivo específico fue el análisis de artículos y manuales sobre precauciones de aislamiento para concluir si existe o no una estandarización en los procedimientos de enfermería durante la práctica diaria, ante la observación de discrepancias en el manejo del material requerido para la aplicación de dichas precauciones en las personas con enfermedades transmisibles, lo cual podría influir en el índice de infecciones nosocomiales.

\section{METODOLOGÍA}

Se realizó una revisión exhaustiva de doce artículos científicos y diez manuales de procedimientos de diferentes instituciones de salud que pudieran dar un sustento teórico a nuestro análisis.

1. Manual de Aislamiento Intrahospitalarios MINSA - Lima Perú; 2003, p.14. Disponible en: http://www.dge.gob.pe/influenza/AH1N1/doctec/ manual_aislamiento_hosp.pdf revisado febrero 2014.

2. ibid.

3. Rubio T, García de Jalón J, Sanjuan F, Erdozain M.A, Sainz de Murrieta J.I, Escobar E, Control de infección. Precauciones estándar. Política de aislamientos, ANALES Sistema Sanitario de Navarra 2000:23, Suplemento 2, p 112. Disponible en: http://es.scribd.com/doc/86314857/Control-deInfeccion- Precauciones-Estandar-Politicas-de-Aislamientos revisado febrero 2014.
Con el apoyo de medios electrónicos también se consultaron las páginas oficiales de la Organización Mundial de la Salud (OMS), la Organización Panamericana de la Salud (OPS) y la Comisión Permanente de Enfermería (CPE), así como la Ley General de Salud, las Normas Oficiales Mexicanas en materia de salud y dos libros de legislación. Se dio una mayor importancia a las fuentes nacionales dado que nos permiten obtener un panorama de lo existente en nuestro país. Se descartaron todos los documentos y artículos que no tuvieran validación teórica. Se estableció la diferencia entre las precauciones que se deberán adoptar con los pacientes en general y las precauciones relacionadas con el aislamiento y las precauciones basadas en la transmisión, lo que nos llevó a la conclusión y propuesta de una mejora.

\section{MARCO TEÓRICO}

El concepto de aislamiento para personas con enfermedades transmisibles fue aplicado desde tiempos bíblicos, como en las colonias de leprosos. ${ }^{4}$ Antes de 1850, las infecciones cruzadas eran comunes y responsables de una alta mortalidad; durante la guerra de Crimea, Florence Nightingal concluyó en la necesidad de dividir a las salas comunes en cubículos y enfatizó la importancia de la asepsia y de mantener los ambientes limpios, cambiando así el concepto de la transmisión de las infecciones. ${ }^{5}$

A inicios de 1889, los hospitales empezaron a usar ambientes de aislamiento individual y agregaron medidas como la utilización de utensilios individuales y el uso de desinfectantes. Grancher en París, Francia, promovió la teoría de la transmisión de enfermedades por contacto, más que por aire, de muchas enfermedades, lo que permitió que pacientes con enfermedades contagiosas fueran internados en salas comunes pero con el uso de separadores de ambiente (biombos). ${ }^{6}$ Esto sirvió para que el personal médico recordara usar guantes y lavarse las manos.

En 1910 surgen las "barreras de enfermería" que establecían un estricto uso de medidas diversas para prevenir la adqui-

4. Manual de Aislamiento Intrahospitalarios MINSA, op. cit.

5. Rubio T, García de Jalón J, Sanjuan F, Erdozain M.A, Sainz de Murrieta J.I, Escobar E, op. cit.

6. Manual de Aislamiento Intrahospitalarios MINSA, op. cit., p. 15. 
sición de infecciones dentro de los hospitales, tales como el lavado de manos con soluciones antisépticas después del contacto y desinfección de materiales contaminados?

El Centro de Control de Enfermedades de Estados Unidos (CDC) inició el desarrollo de las primeras recomendaciones sobre aislamiento en 1967 y las publicó en 1970. ${ }^{8}$ En 1985 y 1986, publicó las recomendaciones para prevenir la infección por VIH en el medio laboral: "Las precauciones universales" (sangre y fluidos corporales), siendo usadas como base de algunas guías publicadas por la OMS con el propósito de adecuarlas a países con menos recursos. ${ }^{9}$ En 1996, el Comité Asesor de Prácticas sobre el Control de Infecciones Hospitalarias (HICPAC) publicó la "Guía para las Precauciones de Aislamiento en las instituciones de hospitalización", fusionando las principales características de las precauciones universales y el aislamiento de substancias corporales -en lo que ahora conocemos como precauciones estándar-para ser utilizadas con todos los pacientes en todo momento e incluyó tres categorías de precauciones basadas en la transmisión (aérea, por gotas y de contacto). ${ }^{10}$

Ahora bien, las personas con enfermedades transmisibles son valoradas para mantenerse en aislamiento; este concepto consiste en la separación de personas infectadas de los huéspedes susceptibles durante el periodo de transmisibilidad de la enfermedad en lugares y bajo condiciones tales que eviten o limiten la transmisión del agente infeccioso, es decir, que en todo paciente que se sospeche o documente un proceso infeccioso con riesgo de transmisión es recomendable una habitación individual con un lavabo de manos, jabón y toallas desechables dentro de la habitación. ${ }^{11}$

La Organización Mundial de la Salud documenta que las infecciones nosocomiales son una importante causa de morbilidad y mortalidad, por lo que la prevención y control de éstas están basadas principalmente en todas aquellas medidas que impiden que el agente infeccioso entre en contacto con el huésped susceptible. ${ }^{12}$

\section{PRECAUCIONES ESTÁNDAR}

Son un conjunto de técnicas para el manejo de líquidos y fluidos o tejidos de todos los pacientes por parte del personal de salud que se fundamentan en el concepto de que todos

7. Rubio T, García de Jalón J, Sanjuan F, Erdozain M.A, Sainz de Murrieta J.I, Escobar E., op.cit., p. 109.

8. Ponce de León R. Baridó M, Rangel F, Soto JL, Wey B S, Zaidi J. Manua de prevención y control de infecciones hospitalarias. Washington DC: OPS;1995:56.

9. Manual de Aislamiento Intrahospitalarios MINSA, op. cit., p. 17.

10. Rubio T, García de Jalón J, Sanjuan F, Erdozain M.A, Sainz de Murrieta J.I, Escobar E, Control, op. cit., p. 112.

11. Ponce de León R. op. cit., p. 49.

12. Ponce de León R. op. cit., p. 450. deben ser considerados como potencialmente infectantes por el virus de la inmunodeficiencia humana u otros agentes infecciosos transmitidos por sangre y fluidos corporales. Éstas se aplican a todos los pacientes que reciben una atención hospitalaria, sin importar su diagnóstico o estado presumible de infección. ${ }^{13}$

- Lavado de manos. Tiene como objetivo eliminar los microorganismos patógenos de la piel, siendo la medida más sencilla y menos costosa demostrada para la prevención de infecciones nosocomiales. ${ }^{14}$

- Uso de cubrebocas. La técnica de colocación de cubre bocas es la acción que se realiza para cubrir la nariz y la boca con una mascarilla para disminuir el riesgo de transmitir microorganismos patógenos al aire, proteger al paciente de infecciones por gotas y prevenir las infecciones adquiridas por la vía aérea. ${ }^{15}$

- Uso de bata. Es el procedimiento que se realiza para la colocación de una bata como barrera de protección para evitar la contaminación de superficies limpias con superficies contaminadas y para proteger la ropa que está en contacto con un paciente infectado o proteger a un paciente con inmunosupresión. ${ }^{16}$

- Uso de guantes. Se utiliza principalmente para reducir los riesgos de colonización transitoria de gérmenes del personal y de éstos a los pacientes; los guantes deben estar limpios y ser desechables. Éstos se usarán cuando exista la posibilidad de contacto con sangre, fluidos orgánicos, secreciones, membranas mucosas, piel no intacta o después de tener contacto con material contaminado; deberán cambiarse los guantes entre los procedimientos en un mismo paciente y entre un paciente y otro. ${ }^{17}$

- Uso de protección ocular. Se utilizará en caso de riesgo de salpicaduras a los ojos; se recomienda para la protección de mucosa conjuntival, durante los procedimientos que puedan ocasionar salpicaduras de sangre o fluidos corporales.

\section{PRECAUCIONES BASADAS EN LA TRANSMISIÓN}

La transmisión de infecciones requiere de tres elementos: una fuente de microorganismos que infecten, un huésped

13. Espinosa de los Monteros M, Solache Alcaraz G, Alamillo García R. Manual de aplicación de precauciones de aislamiento, departamento de epidemiologia, Hospital General de México 2012: p. 12 disponible en http://201.161.5.235/ pdf/pci/manual_precauciones_aislamiento.pdf revisado febrero 2014.

14. Ponce de León R., op. cit., p. 52.

15. Programa de prevención de educación sobre medidas de prevención y control de infecciones nosocomiales para personal de salud, familiares y visitantes, Instituto Nacional de Pediatría, México D.F, 2010: p. 12. Disponible en: http:// www.pediatria.gob.mx/pci_progra.pdf revisado febrero 2014.

16. ibid.

17. Ponce de León R., op. cit., p. 53. 
susceptible y un medio de transmisión. Las fuentes humanas de los microorganismos infectantes pueden ser los pacientes, el personal de salud o en ocasiones, los visitantes y objetos inanimados del ambiente que se han contaminado, incluyendo el equipo.

Éstas son precauciones diseñadas sólo para el cuidado de pacientes específicos que se sabe o sospecha que están infectados o colonizados con patógenos importantes epidemiológicamente que pueden ser transmitidos. ${ }^{18}$

Hay tres tipos de precauciones según su transmisión: precauciones por transmisión aérea, precauciones por transmisión por gotas y precauciones por transmisión por contacto. Éstas pueden combinarse en enfermedades que tienen múltiples vías de transmisión. Cuando se utilizan, ya sean solas o en combinación, deben utilizarse además de las precauciones estándar.

- Precauciones por transmisión aérea. Se utilizan para pacientes confirmados o con sospecha de infección de microorganismos transmitidos por núcleos de gotas transportadas por el aire $(<5 \mu \mathrm{m})$, que pueden dispersarse por corrientes de aire a gran distancia y / o permanecer en suspensión largos periodos de tiempo. ${ }^{19}$

Se debe colocar al paciente en una habitación individual que tenga presión aérea negativa en relación con las áreas circundantes y una adecuada salida de aire al exterior o filtrado de alta eficiencia del aire de la habitación. Si esto no es posible, se debe mantener la puerta de la habitación cerrada y al paciente dentro de ella. Cuando no se disponga de un cuarto privado, se debe colocar en un cuarto con otro(s) paciente(s) que tenga(n) infección activa por el mismo microorganismo, pero no con otra infección. Utilizar protección respiratoria con cubrebocas de alta eficiencia (respirador N95). Limitar el movimiento y transporte del paciente desde su cuarto al exterior; si esto no es posible colocarle un cubrebocas. $^{20}$

- Precauciones para transmisión por gotas. Deberán instalarse en pacientes con infecciones por microorganismos transmitidos por gotas $(>5 \mathrm{mc})$ y que se generan al estornudar, toser, hablar o durante la realización de los procedimientos. Instalar al paciente en un cuarto privado. Si no hay un cuarto privado se hará en cuarto doble

18. Fariñas Álvarez $C$, Teira Cobo $R$, Rodríguez Cundín $P$, Infección asociada a cuidados sanitarios (infección nosocomial), Cantabria. España. Medicine. 2010;10(49), p.3298. Disponible en: http://uiip.facmed.unam.mx/deptos/ microbiologia/pdf/Inf_urinaria_sanitarios_Medicine2010.pdf revisado febrero de 2014.

19. Ponce de León R., op. cit., p. 52.

20. Ibid. con otro paciente del mismo padecimiento, si no hay riesgo de otra infección. Si lo anterior no es posible, se debe mantener una distancia mínima de un metro entre el paciente infectado y otros pacientes o visitantes, así como usar cubrebocas cuando se trabaje muy cerca del paciente (menos de un metro). Se debe limitar el transporte del paciente a lo esencial y en caso de traslado, colocarle cubrebocas, guantes y bata. Éstas se usan si hay riesgo de salpicadura de secreciones respiratorias; los artículos contaminados deben ser desinfectados y/o esterilizados. $^{21}$

- Precauciones para la transmisión por contacto. Se usarán para pacientes específicos con sospecha o diagnóstico de infección o colonización con microorganismos epidemiológicamente importantes, transmitidos por contacto directo con el paciente (contacto con las manos o de piel-a-piel que ocurre cuando la atención del paciente requiere tocar su piel seca) o contacto indirecto con superficies ambientales o artículos del paciente, así como su ropa de cama. ${ }^{22}$

Deberán usarse guantes al entrar al cuarto (los guantes limpios no estériles son adecuados), cambiarse los guantes durante la actividad con el paciente después de tener contacto con el material infectante que pudiera contener concentraciones elevadas de microorganismos (materia fecal, drenaje de heridas), quitárselos antes de salir del cuarto y lavarse las manos inmediatamente, asegurándose que después de esto no se toque ninguna superficie o artículo potencialmente contaminado para evitar así, el transporte del microorganismo fuera del cuarto hacia otras áreas o pacientes. ${ }^{23}$

$\mathrm{Si}$ es inminente el contacto del paciente con la ropa del profesional de salud, se debe colocar una bata limpia no estéril antes de ingresar a la habitación. Asegurarse que su ropa no entre en contacto con las áreas de riesgo después de retirar la bata; depositar la ropa utilizada en una bolsa preparada antes de salir de la habitación y, en la medida de lo posible, el equipo de trabajo rutinario y otros objetos como el estetoscopio, esfigmomanómetro, termómetro y los utensilios de eliminación deben ser para uso exclusivo del paciente. ${ }^{24}$

Es muy clara la necesidad de considerar a todos los pacientes como potencialmente infectantes, pero la

21. Cifuentes Díaz M. Norma de precauciones basadas en la Transmisión (Norma de aislamiento), Hospital clínico Universidad de Chile, 2012 Versión 1: p. 6. Disponible en: http://www.diresacusco.gob.pe/inteligencia/ epidemiologia/guias/guia\%20aislamiento\%20hospitalario.pdf revisado febrero 2014.

22. Fariñas Álvarez C, Teira Cobo R, Rodríguez Cundín P., op. cit. p 3229.

23. Espinosa de los Monteros M, Solache Alcaraz G, Alamillo García R, op. cit., p. 19-21.

24. Ibid. 
utilidad de las precauciones universales se limita a los patógenos transmitidos por sangre. Entonces es cuando se decide realizar una combinación del aislamiento para substancias corporales y precauciones universales, resultando las "Precauciones estándar", diseñadas para reducir el riesgo de transmisión de patógenos transmitidos por sangre y también por otros patógenos. Este tipo de recomendaciones deberán ser utilizadas en todos los pacientes hospitalizados. ${ }^{25}$

Dado que el CDC considera que es muy difícil que estas recomendaciones satisfagan las características de los más de 6,000 hospitales norteamericanos y más difícil aún, que lo hagan para los más de 15,000 hospitales latinoamericanos, se recomienda que los hospitales revisen las guías y las adapten de acuerdo con su infraestructura y recursos existentes de una manera práctica y prudente. ${ }^{26}$

\section{CONCLUSIONES}

Como resultado de la revisión de diferentes artículos, se ha verificado la importancia de las precauciones de aislamiento en pacientes con enfermedades transmisibles, en una institución de tercer nivel y se ha comprobado que sí existe una estandarización en las normas recomendadas para el aislamiento del enfermo infeccioso. Sin embargo, con la revisión de los manuales de diferentes instituciones pudimos darnos cuenta que hace falta ser más especifico en el uso y manejo del material requerido para cumplir con las recomendaciones para el aislamiento, ya que un número importante de estos manuales no cuenta con estos datos.

Las precauciones estándar están diseñadas para reducir el riesgo de transmisión de microorganismos tanto de fuentes conocidas como desconocidas de infección entre los pacientes y los trabajadores dentro de los hospitales. El lavado de manos y la práctica de las precauciones estándar protegen de los agentes infecciosos a los pacientes, al personal de la salud y a todo aquel individuo que tenga contacto con el medio hospitalario, por tanto, deben ser base de toda terapéutica intrahospitalaria.

Debido a que los recursos siempre serán limitados, es necesario planear cuidadosamente un sistema que permita asegurar el abastecimiento del personal, los materiales y los equipos esenciales. Aunado a una difusión detallada de los lineamientos para adoptar dichas precauciones por medio de manuales específicos. El éxito de esta implementación depende de una organización correcta de los

25. Ponce de León R., op. cit., p. 55

26. Ponce de León R., op. cit., p. 51. conocimientos, creatividad, disposición y difusión en el equipo de salud.

\section{LECTURAS RECOMENDADAS}

— Álvarez Tuñón $\mathrm{Z}$ et al. Guía de aislamiento para pacientes con infecciones transmisibles edición resumida [Internet]. Consejería de Salud y Servicios Sanitarios Hospital Valle del Nalón Asturias; 2007 [acceso febrero de 2014]: 40. Disponible en: http://www.hca.es/huca/web/contenidos/servicios/dirmedica/almacen/preventiva/Gu\%C3\%ADa\%20 aislamiento\%20Resumida.pdf

- Biblioteca virtual de la OPS. Biblioteca virtual en salud/descriptores de la ciencia de la salud, ID: 029750 [Internet]. [Acceso 14 de febrero 2014]. Disponible en: http://pesquisa.bvsalud.org/portal/resourse/es/029750.

- Cuervo Polanco MP. Técnicas para el aislamiento hospitalario, Guías para el manejo de urgencias. Bogotá Colombia: 1426-432. [acceso febrero de 2014]. Disponible en: http://www.aibarra.org/Apuntes/criticos/Guias/ Enfermeria/Tecnicas_para_el_aislamiento_hospitalario.pdf

- Chi J. El control de las enfermedades transmisibles. Informe Oficial de la Asociación Estadounidense en Salud Pública OPS. Publicación Científica y Técnica No. 581. 17 a ed. Washington, D.C.; 2001[acceso febrero 2014]: 673. Disponible en: http://iris.paho.org/xmlui/bitstream/ handle/123456789/739/9275315817.pdf?sequence=1

- Damián Yáñez L, Manual de procedimientos técnicos del departamento de enfermería. México: Instituto Nacional de Enfermedades Respiratorias, Ismael Cosío Villegas: 2009 [acceso febrero 2014]: 378. Disponible en: http://www.iner.salud.gob.mx/descargas/manuales/manuales_procedimiento/dirmedica/enfermeriatec.pdf

- Enzo Latorre A. Recomendaciones y actualización de la normativa de aislamiento de pacientes del programa de infecciones intrahospitalarias República de Chile. Circular 46. Ministerio de Salud. División de Salud de las personas, programa de Infecciones Intrahospitalarias. 1998 [acceso febrero 2014]: 9. Disponible en: http://info.seremisaludatacama.cl/documents/Autorizacion_Sanitaria/Tramites\%20Sanitarios/ Unidad\%20de\%20Profesiones\%20M\%C3\%A9dicas/Normas\%20y\%20 Reglamentos\%20A.S/Profesiones\%20M\%C3\%A9dicas/Infecciones\%20 Asociadas\%20a\%20Atenci\%C3\%B3n\%20de\%20Salud\%20-\%20IAAS/ CIRCULAR\%2046\%20Actualizci\%C3\%B3n\%20Aislamiento\%20 MINSAL.pdf

- Gómez F, Ochoa Linares M, Gonzáles Zarate. Guía de precauciones de aislamiento hospitalario Cusco. 2006 [acceso febrero 2014]: 23. Disponible en: http://www.diresacusco.gob.pe/.../guias/GUIA\%20AISLAMIENTO $\% 20 \mathrm{HO}$

- Guirao-Goris JA, Olmedo Salas A, Ferrer Ferrandis E. El articulo de revisión. Revista Iberoamericana de Enfermería comunitaria. 2008 [acceso febrero 2014]; 1: 25. Disponible en: http://revista.enfermeriacomunitaria. org/articuloCompleto.php?id=7

- Hernández Valdez E, Acosta González M, Nadal Tur B, Pijuan Pérez M, Fon Abreu Y, Armas Rojas N. Intervención educativa para incrementar los conocimientos sobre bioseguridad en el personal de enfermería de una institución hospitalaria [internet]. Revista Cubana Enfermera. 2006; 22 (2). ISSN 1561-2961. Disponible en http://bvs.sld.cu/revistas/enf/ vol22_2_06/enf08206.htm revisado febrero 2014.

- Infecciones hospitalarias, legislación en América Latina. Documento técnico- HDM/CD/hq/500-07. Disponible en: http://www.org/hq/index. php?option=com_joomlabook\&jtemid=260\&jang=es

- Ley General de Salud de los Estados Unidos Mexicanos. Última modificación, enero 2014. Disponible en: Http://www.gparlamentario.org/.../ Ley\%20General\%20de\%20Salud.pdf. (febrero 2014):

- Ade y Torrent MP, Bolis M. Infecciones hospitalarias, legislación en América Latina. Documento técnico- HDM/CD/hq/500-07. Washington D.C.: OPS: 2007 [acceso febrero 2014]. Disponible en: http://www.org/ hq/index.php?option=com_joomlabook \&jtemid=260\&jang $=e s$

- Políticas para el aislamiento para pacientes con enfermedades transmisibles, Departamento de infectología, Comité de infecciones intrahospi- 
talarias. México D.F.: Instituto Nacional de Neurología y Neurocirugía MVS; 2013: 14.

- Políticas de Precauciones en el personal de salud y visitantes del Instituto Nacional de Neurología y Neurocirugía MVS, Departamento de infectología, Comité de infecciones intrahospitalarias. México D.F.: Instituto Nacional de Neurología y Neurocirugía MVS; 2013: 5.

- Precauciones y aislamientos ante patología infecciosa. Servicio de M. Preventiva del Hospital Ramón y Cajal de Madrid. 2002. disponible en: http://www.saludcastillayleon.es/HSReyesAranda/es/calidad/ precauciones-aislamientos-patologia-infecciosa

- Requisitos de Uniformidad para Manuscritos Presentados a Revistas Biomédicas (Estilo Vancouver)

- Rodríguez MI, Espinoza E, Menjívar EV, Lineamientos para el control de infecciones de atención sanitaria. El Salvador: Editorial del Ministerio de Salud Pública; 2010 [acceso febrero 2014]. Disponible en: http://www.paho.org/els/index.php?option=com_docman\&tash=doc_ dowland $\&$ gid $=890 \&$ itemid=99999999-969K 